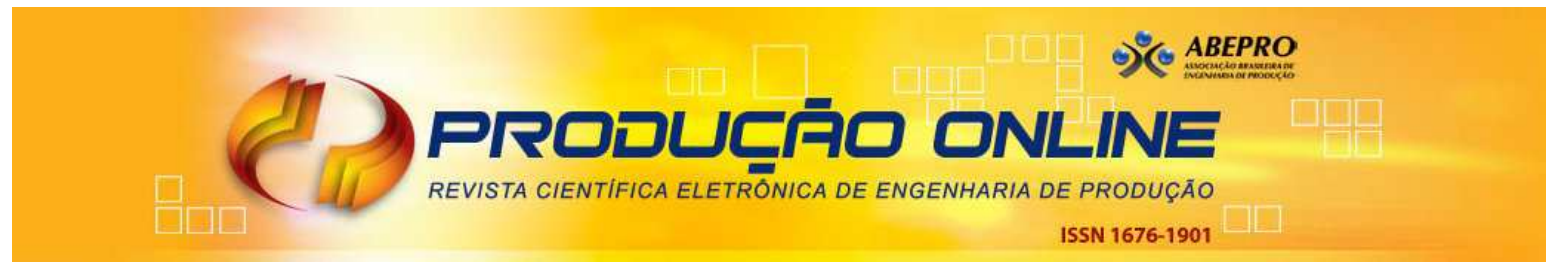

\title{
UM MODELO DE SIMULAÇÃO DE SISTEMAS APLICADO À PROGRAMAÇÃO DA PRODUÇÃO DE UM FRIGORÍFICO DE PEIXE
}

\section{A SYSTEM SIMULATION MODEL APPLIED TO THE PRODUCTION SCHEDULE OF A FISH PROCESSING FACILITY}

\author{
Carla Roberta Pereira* E-mail: pereiracrz@gmail.com \\ Miguel Antonio Bueno da Costa* E-mail: mbcosta@ufscar.br \\ *Universidade Federal de São Carlos (UFSCar), São Carlos, SP
}

\begin{abstract}
Resumo: A simulação busca importar a realidade para um ambiente controlado, onde se pode estudar o seu comportamento, sob diversas condições, sem riscos físicos e/ou grandes custos envolvidos. Dessa forma, ela se torna uma técnica útil e poderosa em mercados crescentes, como é o caso da tilapicultura que necessita produzir de maneira adequada para atender bem seus clientes e expandir seus negócios. Nesse sentido, o objetivo central deste trabalho foi desenvolver um modelo de simulação para auxiliar na tomada de decisões sobre a programação da produção de um frigorífico de peixe. Para tanto, utilizou-se como método de pesquisa o estudo de caso e a modelagem/simulação, incluindo neste conjunto a metodologia SimuCAD e as etapas de desenvolvimento de um modelo de simulação. Com a construção do modelo foi possível trabalhar diversas alternativas de cenários, testando diferentes jornadas de trabalho, tipos de fluxos e de capacidade produtiva, além de variações do estoque final com relação às vendas. Como resultado, obteve-se um modelo de simulação útil e diferenciado para auxiliar na tomada de decisão sobre a programação da produção do frigorífico de peixe estudado.
\end{abstract}

Palavras-chave: Simulação. Programação da produção. Frigorífico de peixe. Tilapicultura. Software Arena.

\begin{abstract}
The simulation seeks to import the reality to a controlled environment, where it is possible to study it behavior, under several conditions, without involving physical risks and/or high costs. Thus, the system simulation becomes a useful and powerful technique in emergence markets, as the tilapiculture sector that needs to expand its business. The main purpose of this study was the development of a simulation model to assist the decisions making of the production scheduling of a fish processing facility. It was applied, as research method, the case study and the modeling/simulation, including in this set the SimuCAD methodology and the development phases of a simulation model. The model works with several alternative scenarios, testing different working shifts, types of flows and production capacity, besides variations of the ending inventory and sales. The result of this research was a useful and differentiated model simulation to assist the decision making of the production scheduling of fish processing facility studied.
\end{abstract}

Keywords: Simulation. Production Scheduling. Fish Processing Facility. Tilapiculture. Software Arena. 


\section{INTRODUÇÃO}

A melhoria da qualidade dos produtos, a redução de custos operacionais e a inovação tecnológica são aspectos importantes para sustentar as estratégias das organizações na disputa por mercados. Para assegurarem lucro no médio e longo prazo, necessitam criar e gerenciar conhecimentos, obtendo vantagens reais ao desenvolver os seus sistemas de produção e oferecendo produtos e/ou serviços adequados às necessidades de seus clientes.

A falta de informações, a inexistência de situações análogas passadas, a complexidade associada aos problemas identificados, tudo faz com que a organização procure por métodos e técnicas que a orientem na busca por soluções. Nesse contexto, a Pesquisa Operacional, através da Simulação de Sistemas, pode contribuir de forma efetiva (GAVIRA, 2003).

A simulação é útil na resolução de problemas complexos que podem envolver situações determinísticas ou estocásticas. Assim, Morabito e Pureza (2010, p.170) definem simulação como "uma importante ferramenta de planejamento que procura emular, por meio de relações lógicas, o funcionamento de sistemas reais, a fim de observar seu comportamento sob diferentes cenários".

Os investimentos com modificações de produtos, processos, tecnologias e arranjos físicos são altos e arriscados. A simulação permite uma visualização mais detalhada do funcionamento da planta e ainda testes com cenários alternativos para indicar soluções a baixo custo, através de modelos computacionais.

Nesse contexto, o objetivo central desse trabalho foi desenvolver um modelo de simulação para auxiliar na tomada de decisões sobre a programação da produção de um frigorífico de peixe, localizado no interior do estado de São Paulo - Brasil. Utilizouse como ferramenta computacional o software Arena, e como método de pesquisa, a modelagem/simulação e o estudo de caso, incluindo a metodologia SimuCAD e as etapas de desenvolvimento de um modelo de simulação discutidas por Banks (1998) e Law e Kelton (2000). 
O artigo se estrutura em cinco partes. A primeira expõe uma abordagem teórica sobre assuntos fundamentais envolvidos nesta pesquisa, sendo eles: simulação de sistemas, software Arena, sistemas de produção, planejamento, programação e controle da produção e uma breve abordagem sobre a tilapicultura. A segunda apresenta a método de pesquisa utilizado. A terceira e a quarta descrevem o desenvolvimento e a análise dos resultados da pesquisa. Por fim, a quinta parte comenta e conclui o trabalho.

\section{REVISÃO TEÓRICA}

De acordo com Cauchick Miguel et al. (2010), o referencial teórico delimita as fronteiras do que será investigado, proporciona suporte teórico à pesquisa, explicita o grau de evolução (estado da arte) do tema estudado, além de ser um indicativo de familiaridade e conhecimento sobre 0 assunto para o pesquisador. Com essa preocupação, foi feita uma revisão da literatura que contempla os temas tratados neste estudo.

\subsection{Simulação de Sistemas}

Com um mercado cada vez mais competitivo e de mudanças rápidas, a simulação tem-se tornado uma ferramenta útil e poderosa à análise de sistemas complexos (HARREL; TUMAY, 1995) e à resolução de problemas, indispensáveis aos engenheiros, projetistas e administradores (SHANNON, 1998). Ela analisa problemas e serve como ferramenta de análise de sistemas complexos reais ou sob condições específicas determinadas pelo usuário (COSTA, 2002; UM; HYEONJAE; LEE, 2009).

Segundo Pegden, Shannon e Sadowski (1995), a simulação é o processo de projeção de um sistema real a um modelo relativo que, a partir deste, conduz experimentos a fim de entender seu comportamento e/ou avaliar estratégias de operação. Sendo assim, é possível importar a realidade para um ambiente controlado, 
onde se pode estudar o seu comportamento sob diversas condições, sem riscos físicos e/ou grandes custos envolvidos (FREITAS FILHO, 2001).

De forma geral, a simulação e a modelagem podem ser consideradas técnicas integradas, sendo necessário o desenvolvimento de um modelo para posteriormente simulá-lo (ÖREN, 2010). Normalmente, os modelos incorporam várias entradas (parâmetros) no sistema, como tempo, distância, velocidade e recursos disponíveis, e fornecem um meio estatisticamente válido para avaliar, redesenhar e quantificar a utilização de recursos, a racionalização de processos e o tempo gasto (HARRINGTON; TUMAY, 2000; LÖFGREN; TILLMAN, 2011).

As informações geradas auxiliam nas tomadas de decisão e contribuem para uma melhor compreensão do sistema estudado (COSTA, 2002). Esta técnica ainda permite o tratamento estocástico das variáveis do processo, o que assegura uma representação mais realista da aleatoriedade do sistema, diferentemente se tratadas apenas com valores médios destas variáveis (OLIVEIRA, 2008).

Lobão e Porto (1996) e Ören e Yilmaz (2012) afirmam que a simulação dispõe de uma série de inferências sobre as mais diversas atividades nos sistemas de produção, tais como: identificação de problemas; comparação com o desempenho de outros sistemas; estudos sobre a utilização da capacidade instalada, níveis de inventário, lógica de controle, projetos de produto e engenharia, integração, seqüenciamento, gargalos do sistema, melhor arranjo físico e melhor índice de produtividade dos funcionários; treinamento de operadores; análise de sensibilidade; auxílio ao planejamento e a aquisição; e previsão de comportamento e desempenho;

Kelton, Sadowski e Sadowski (1998) afirmam que a simulação computacional iniciou na década de 80 e, desde então, tem sido amplamente utilizada tanto no meio acadêmico quanto no empresarial, graças ao aumento da popularidade e da capacidade dos computadores. Assim, várias são as maneiras de se desenvolver um modelo computacional, incluindo desde as linguagens de alto nível até planilhas ou aplicativos de software (ALEXANDER, 2006). Diversos simuladores de fácil utilização e acompanhados de animação gráfica dinâmica do sistema estão disponíveis no mercado como, por exemplo, o Arena, o AutoMod e o ProModel. 
A simulação tem sido aplicada em muitos setores, como o hospitalar (WANG et al., 2010) o aeroportuário (MEDAU, 2011), o sucroalcooleiro (IANNONI; MORABITO, 2002; SILVA, 2006), o agropecuário (COSTA, 2004), o de transporte (WEI; LEUNG, 2011) e principalmente o de manufatura (WY et al., 2011). Em indústrias frigoríficas, poucas foram às aplicações encontradas, sendo as mais recentes aquelas ligadas ao abate de carne bovina, suína e avícola. Com relação ao processo produtivo ligado ao objeto de pesquisa deste estudo - frigorífico de peixe - identificou-se apenas os trabalhos de Jonatansson e Randhawa (1986), Jensson (1988) e Randhawa e Bjarnanson (1995). Nenhum destes, no entanto, utilizou softwares do tipo Arena para análise do processo.

Esta técnica, portanto, é considerada muito útil ao oferecer segurança na minimização de riscos e custos com diversos recursos e ações (SHANNON, 1998). Contudo, ela não é uma ferramenta que substitui o trabalho de interpretação humano (DUARTE, 2003), pois ao investigar uma dada situação problema com alta variabilidade das variáveis, gera resultados que demandam interpretações mais profundas e abrangentes (PAUL; KULJIS, 2010).

\subsection{Software Arena}

Lançado pela Systems Modeling Company em 1993, o Arena é um dos softwares de simulação mais utilizados no mundo. Este, além de possibilitar o desenvolvimento de diversos modelos (lógica) e cenários (animação) de produção, envolvendo chegada de matéria-prima, quantidade de funcionários, tempos de processo, gargalos e mix de produto, também contém um conjunto de aplicações embutidas (Input Analyser, Output Analyser e OptQuest) que auxilia na análise estatística dos dados de entrada e de saída e na identificação de um resultado "ótimo" (KELTON; SADOWSKI; SWETS, 2010).

Como outros simuladores atuais e com auxílio de equipamentos computacionais mais avançados, o Arena fornece um pacote com (GAVIRA, 2003, p.85): interface gráfica de comunicação com o usuário; animação do modelo; relatórios em tempo real sobre a simulação executada; coleta automática de saídas para medição de 
desempenho do sistema; desenvolvimento de modelos orientados a objetos; e ferramentas estatísticas para tratamento dos dados da simulação.

\subsection{Sistemas de Produção}

Segundo Sipper e Bulfin (1997), os sistemas de produção são como icebergs, onde a parte visível (geralmente os processos de transformação) é ínfima perto do todo. Desta forma, Fernandes e Godinho (2010, p.1) o define como "um conjunto de elementos (humanos, físicos e procedimentos gerenciais) inter-relacionados que são projetados para gerar produtos finais cujo valor supere o total dos custos incorridos para obtê-los". Tais sistemas são compostos de entradas (inputs), processo de transformação, saídas (outputs) e de um subsistema de realimentação (feedback) (BOIKO; TSUJIGUCHI; VAROLO, 2009; LI; MEERKOV; ZHANG, 2010).

Sipper e Bulfin (1997) tipificam os sistemas de produção, de acordo com o fluxo do processo, em: sistemas por projeto (Project Shop), sistemas intermitentes (Job Shop), sistemas por lotes (Batch Shop) e sistemas de fluxo contínuo (Flow Shop). Os mesmos autores classificam o tipo do arranjo físico de uma planta com relação ao volume e variedade dos produtos, sendo como: layout por posição fixa, layout por processo, layout por produto e layout por célula.

A classificação dos sistemas é de grande importância nas organizações, pois permite: um melhor entendimento do sistema observado; o estabelecimento de relações entre as características do sistema; a seleção de ferramentas de análise; o entendimento de problemas típicos e suas soluções particulares; bem como a seleção de técnicas de Planejamento, Programação e Controle da Produção, Controle da Qualidade do Processo, Gestão de Fornecedores, Gestão da Demanda e Gestão da Distribuição (BOIKO; TSUJIGUCHI; VAROLO, 2009).

\subsection{Planejamento, Programação e Controle da Produção}

De acordo com Tubino (1997) e Fernandes e Godinho (2010), as atividades de Planejamento e Controle da Produção (PCP) são exercidas em três níveis hierárquicos 
(estratégico, tático e operacional) nas atividades de um sistema de produção. No nível estratégico são definidas políticas que compõem o planejamento estratégico da produção, o qual estabelece um plano de produção de longo prazo, segundo as estimativas de vendas e a disponibilidade de recursos. Já, no nível tático são desenvolvidos planos de médio prazo, definidos como plano mestre da produção. Este plano só é viável se for compatível com as decisões tomadas no nível anterior. Finalmente, no nível operacional são preparados os programas de curto prazo de produção, englobando a programação da produção, a administração dos estoques, a emissão e liberação das ordens de compras, fabricação e montagem, bem como a execução do controle da produção (CHASE; JACOBS; AQUILANO, 2006).

Focando no ponto chave deste estudo dentro da função PCP, a programação da produção ocupa-se da implementação de tudo que foi planejado, porém no curto prazo. De acordo com Nunes, Melo e Nigro (2009), ela consiste em estabelecer, com maior eficiência, um sequenciamento ótimo das ordens de produção para minimizar e otimizar a utilização dos recursos. Sendo assim, a programação define a carga dos centros de produção e os momentos nos quais ela deve ser executada, a fim de cumprir os prazos e programas de entrega assumidos (VOLLMANN et al., 2004). Logo, esta atividade prepara e distribui ordens das mais diversas espécies, cuja finalidade é passar instruções sobre o que, como, onde e quando os diversos setores da fábrica devem operar (CONTADOR; CONTADOR, 1997).

Apesar da frequente confusão entre as funções de planejamento e programação, ambas são consideradas distintas. A primeira está relacionada a projeções gerais e de longo prazo, enquanto que a segunda refere-se ao dia a dia ou horizontes mais restritos (DIRENE, 2003). Por conseguinte, os objetivos da programação, segundo Chase, Jacobs e Aquilano (2006, p.592), são: "cumprir as datas de entrega, minimizar os leadtimes, minimizar custo ou tempo de setup, minimizar o estoque em processo e maximizar a utilização da máquina ou mão de obra”. Contudo, é sempre bom lembrar que a teoria sobre Produção Enxuta ressalta que manter todos os recursos ocupados pode não ser um meio eficaz de se conseguir bons resultados. 


\subsection{Tilapicultura}

Tilápia "é a denominação comum de grande gama de espécies de peixes ciclídeos" (BOSCOLO et al., 2001, p.1391) de origem africana. Por se tratar de um peixe tropical, águas quentes são ideais para a sua reprodução e desenvolvimento. Muitas são as vantagens que tornam as tilápias um grupo de peixes mundialmente cultivado, por exemplo: aceitam uma grande variedade de alimentos; seu ciclo de engorda é relativamente curto (seis meses em média) e a desova ocorre durante todo o ano; são resistentes a doenças, a altas densidades de povoamento e a baixos níveis de oxigênio dissolvido; possuem boas características organolépticas - carne branca de textura firme e saborosa, baixo teor de gordura, ausência de espinhos intramusculares em forma de "Y" - e excelente rendimento de filé (aproximadamente 35\% a 40\%), que a potencializa como peixe para industrialização (HILSDORF, 1995; RESENDE, 2010).

A tilápia, atualmente, é a espécie mais cultivada no território brasileiro e a segunda no mundo (JORY; ALCESTE; CABRERA, 2000). Nos últimos 56 anos, o consumo mundial per capita de pescados dobrou em virtude das mudanças alimentares e dos incentivos a aquicultura (FIGUEIREDO JUNIOR; VALENTE JUNIOR, 2008). A produção de tilápias, no Brasil, teve um grande crescimento e, em 2005, ultrapassou a produção conjunta dos principais países exportadores de filé fresco para o mercado americano (Equador, Honduras, Costa Rica e Colômbia). Assim, com este crescimento acentuado nas exportações de tilápia, principalmente para os EUA e o Canadá, estimulou-se a instalação de unidades de processamento, além de despertar o interesse de investidores nacionais (SUSSEL, 2007; KUBITZA, 2007).

No início, o processamento da tilápia era realizado em escala reduzida em frigoríficos de pequeno porte. Atualmente, as indústrias beneficiadoras vêm crescendo em tamanho e em número. Os produtos resultantes do processamento para alimentação humana podem ser: filés, postas, peixe inteiro eviscerado, hambúrgueres, nuggets, quibes, espetinhos, petiscos, lingüiças, produtos defumados, sashimi e outras variações que atrai o consumidor. Pode-se, também, obter produtos não alimentícios com as carcaças, escamas e vísceras das tilápias, como farinha, ração, óleo; e do 
couro, diversos acessórios como bolsas, sapatos e cintos (KUBITZA, 2004; FIGUEIREDO JUNIOR; VALENTE JUNIOR, 2008).

Apesar do índice de consumo de pescado no Brasil ser baixo, os consumidores atuais têm buscado alimentos que sejam de rápido preparo e com qualidade nutricional, o que incentiva a pesquisa para novos produtos. Assim, a perspectiva é de que em 2030 o consumo seja de $22,5 \mathrm{~kg} /$ pessoa e não mais $7 \mathrm{~kg} /$ pessoa como em 2003 (SOARES, 2005; RESENDE, 2010).

\section{MÉTODO DE PESQUISA}

A pesquisa é caracterizada como um processo formal e sistemático de desenvolvimento do método científico, cujo objetivo fundamental é descobrir respostas para problemas mediante o emprego de procedimentos científicos (GIL, 1999). Assim, de acordo com Cauchick Miguel et al. (2010), os quatro tipos de métodos de pesquisa mais utilizados em Engenharia de Produção e Gestão de Operações são: survey, estudo de caso, pesquisa-ação e modelagem/simulação. Esta pesquisa utilizou dois métodos de pesquisa: o estudo de caso, para conhecer o fenômeno no contexto real de forma ampla e detalhada, e a modelagem/simulação, que aborda quantitativamente o problema e busca controlar as variáveis gerenciais em estudo.

O segundo método busca desenvolver um modelo que de acordo com Morabito e Pureza (2010, p.166) "é um veículo para se chegar a uma visão estruturada da realidade" e, posteriormente, simulá-lo. A metodologia orientadora dos passos desse trabalho foi a metodologia SimuCAD (COSTA; MENEGON; CAMAROTTO, 1996) somada ao projeto de desenvolvimento de um modelo e processo de simulação proposto por Banks (1998) e Law e Kelton (2000).

\subsection{Metodologia SimuCAD}

A metodologia utilizada como auxílio ao estudo do problema e posterior desenvolvimento do modelo de simulação proposto foi desenvolvida por um grupo de 
pesquisa - SimuCAD - do Departamento de Engenharia de Produção da Universidade Federal de São Carlos (UFSCar). Por este motivo, ela é denominada metodologia SimuCAD. Ela comporta três campos distintos do conhecimento: pesquisa-ação, engineering design e simulação. Para esta pesquisa, focou-se a simulação aplicada ao estudo de um sistema de produção.

Os módulos que compõem a metodologia SimuCAD estão inseridos em um sistema maior, denominado "Ambiente", como pode ser visto na Figura 1. O primeiro módulo - Mix de Produto e Tecnologia de Produção - busca identificar no sistema de produção quais os produtos resultantes e o processo de obtenção dos mesmos. O segundo módulo - Fatores de Produção - tem o propósito de identificar e quantificar os recursos diretos e indiretos empregados na produção, atentando para os detalhes do processo e dos produtos. O terceiro módulo - Centros de Produção - trata da representação das demandas espaciais do processo produtivo a partir da descrição dos centros de produção de forma detalhada. Após o conhecimento e a coleta dos dados dos três primeiros módulos, os três seguintes são representados de forma paralela, pois constituem um processo cíclico de construção, verificação e validação do modelo (etapas detalhadas no item 3.2) a partir de cenários estratégicos desejados. Definido o modelo, parte-se para o seu detalhamento e animação.

Figura 1 - Macroestrutura da metodologia SimuCAD

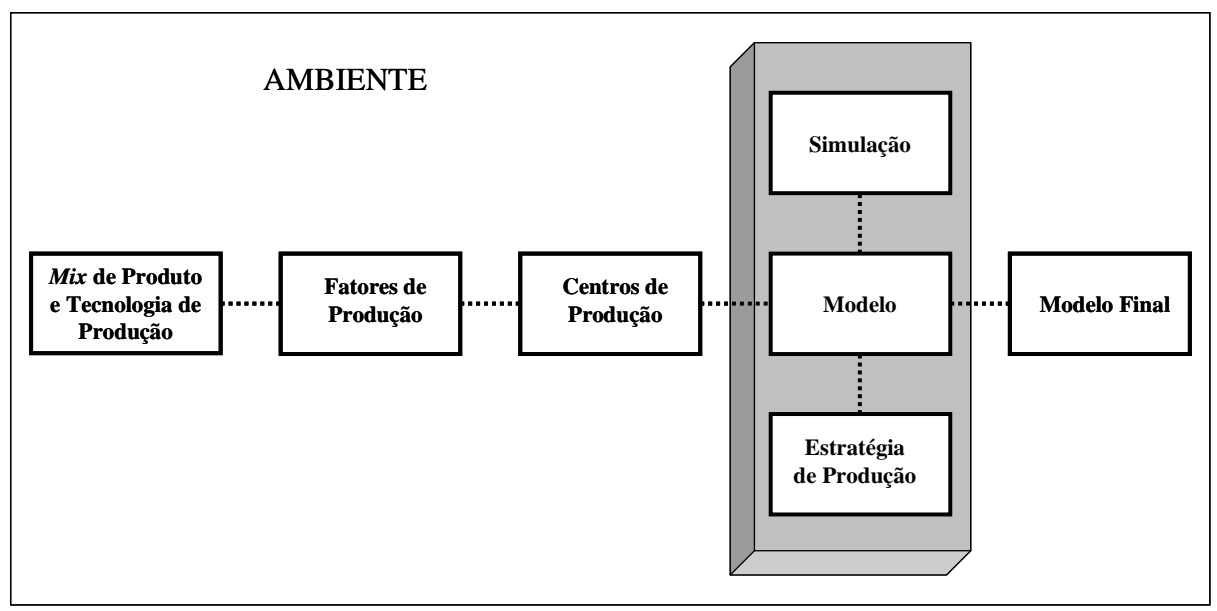

Fonte: Adaptado de Costa; Menegon; Camarotto (1996) 


\subsection{Etapas do Desenvolvimento de um Modelo}

De acordo com Shannon (1975) o desenvolvimento de um processo de simulação é mais uma extensão das artes do que das ciências. Tal afirmação ajuda a entender o porquê da dificuldade de se apresentar um mecanismo único para desenvolver modelos de simulação. Contudo, no geral, há alguns elementos fundamentais de um modelo de simulação e alguns passos que, se seguidos, podem ajudar o "modelista", mesmo novato, a atingir um bom desempenho no desenvolvimento de modelos de simulação (COSTA, 2002). Neste sentido, segundo adaptações de Banks (1998) e Law e Kelton (2000), um projeto de simulação deve seguir os seguintes passos (Figura 2):

Figura 2 - Etapas de um projeto de simulação

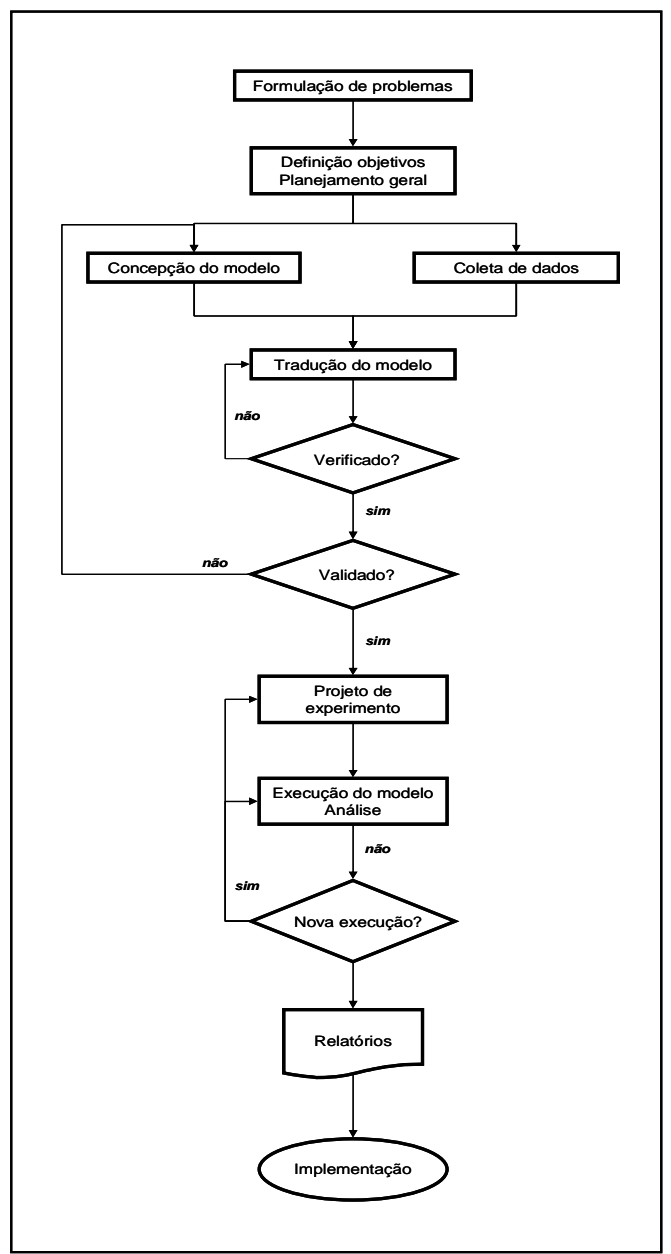

Fonte: (BANKS, 1998, p.16)

Revista Produção Online, Florianópolis, SC, v.12, n. 4, p. 972-1001, out./dez. 2012. 
a) Definição do problema: define-se claramente o problema para estabelecer a finalidade, ou seja, por que estudá-lo?

b) Definição dos objetivos e plano geral do projeto: estabelece-se o(s) objetivo(s) da pesquisa e demais necessidades - o tempo necessário, a equipe utilizada, os requisitos de hardware e software e o custo dos procedimentos.

c) Conceituação do modelo: traduz-se o sistema real para um modelo conceitual, por meio de uma série de relações matemáticas e lógicas sobre componentes e estrutura do sistema (exemplo: fluxograma).

d) Coleta de dados: reúnem-se informações como, fluxos de informações, recursos, procedimentos da operação e considerações teóricas.

e) Tradução do modelo: codifica-se no software de simulação o modelo conceitual tornando-se, assim, um modelo operacional.

f) Verificação: trata-se de realizar testes com o modelo, de modo a verificar se o mesmo foi construído conforme desejado.

g) Validação: verifica-se se modelo é uma boa representação do sistema real e se suas respostas são compatíveis com as saídas do mesmo, dentro de um nível de confiança desejável.

h) Projeto de experimentos: etapa para planejar os conjuntos de variáveis controláveis e parâmetros do modelo que serão utilizados na etapa seguinte.

i) Testes e análises: executa-se o modelo diversas vezes para alcançar resultados confiáveis, com a utilização de abordagens estatísticas para análise dos dados de entrada e de saída.

j) Mais testes?: determina-se se é necessário efetuar mais testes com o modelo ou se eventuais cenários adicionais precisam ser desenvolvidos, a partir das análises que já foram concluídas.

I) Documentação e relatórios: relata-se de forma clara e concisa todos os resultados gerados, pois serão utilizados nas análises e nos processos de futuras tomada de decisão.

m) Implementação: coloca-se em prática as informações e ações descritas no relatório. 


\section{A PESQUISA, O MODELO E O ESTUDO DE CASO}

\subsection{Apresentação da empresa}

O frigorífico de peixe em estudo está a apenas dois anos no mercado. Localizase no interior do estado de São Paulo e faz parte de uma Corporação (Grupo) que consiste em quatro unidades de negócio: frigorífico de peixe, pisciculturas (Unidade I, II e III), fábrica de ração e pecuária. Seu sistema de produção é composto por entradas (matéria-prima, mão de obra e equipamentos), processo (recepção, lavagem e escamagem, beneficiamento, congelamento, embalagem e estoque final) e saídas (filés de tilápia de várias classificações).

A empresa produz sob encomenda (clientes e distribuidores fixos) e para estoque (terceiros), tem baixo nível de contato com o consumidor, um mix de produtos que pode ser considerado de pequeno a médio, e trabalha sob influência da sazonalidade. O arranjo físico predominante deste sistema de produção é o de processo, pois a matéria-prima passa por todos os recursos transformadores de acordo com a sequência estabelecida das operações. Não há uma forma sistematizada de plano de produção no longo prazo. Sendo assim, a única programação existente é com base nos pedidos diários e semanais recebidos pelos distribuidores, clientes fixos e consumidores esporádicos. Geralmente, estes pedidos são feitos no final de semana com prazo de entrega para quarta e quinta-feira. Não existe, formalmente, nenhum roteiro de produção ou previsão de vendas que se oriente por informações históricas, por exemplo. Os estoques são verificados pelos próprios funcionários, sem nenhuma política definida.

\subsection{Desenvolvimento da Pesquisa}

As etapas utilizadas para desenvolvimento desta pesquisa seguiram as indicadas pela metodologia SimuCAD e, quando se fez necessário a geração de um modelo de simulação, seguiu-se os passos do desenvolvimento de um modelo de simulação apresentados por Banks (1998) e Law e Kelton (2000). 
De acordo com a primeira etapa da metodologia SimuCAD definiu-se como mix de produtos os filés de tilápias das seguintes classificações: mini (com peso menor que $40 \mathrm{~g}$ ), 40-80g, 80-120g, 120-180g, 180-250g e premium (com peso maior que 250g). Cada uma destas categorias é classificada, ainda, quanto à porcentagem de água agregada, ocorrida por meio do processo de glasing: A (filé só congelado), B (filé com $8 \%$ de água), C (filé com 15\% de água), D (filé com 30\% de água, e F (filé fresco). Toda a tecnologia de produção foi identificada, aprendida e registrada.

$\mathrm{Na}$ etapa seguinte foram quantificados, orientados pela estrutura do produto, todos os insumos utilizados (recursos diretos) e os colaboradores, máquinas e instrumentos de trabalho (recursos indiretos). Já, na terceira etapa descreveu-se o processo produtivo da planta em estudo (Figura 3) que consiste na chegada e armazenamento dos peixes em câmara fria, passando, posteriormente, pela escamagem, lavagem, e sendo transportados por esteiras até 0 setor de beneficiamento, onde os filés são extraídos. Depois de separado os filés dos couros, carcaças, vísceras e espinhos centrais, eles são classificados em pesos e colocados em bandejas. Essas são posicionadas em carrinhos e levadas até a câmara de congelamento. Depois de congelado, os filés que não passam pelo processo de glasing (agregação de água), são retirados das bandejas, embalados em caixas de $15 \mathrm{~kg}$ e transportados até a câmara fria de produto acabado. Lá permanecem até o momento da expedição. 
Figura 3 - Fluxograma do processo

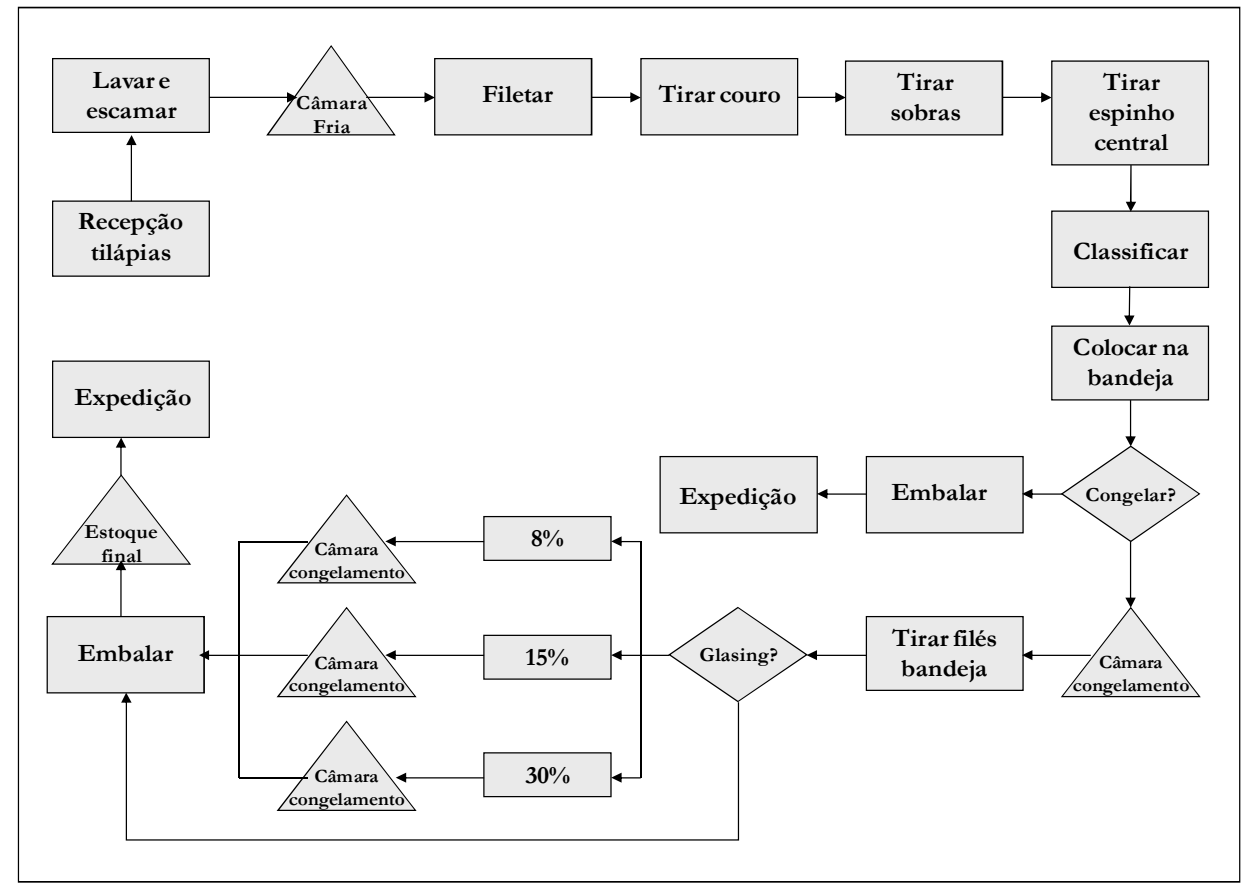

Conforme especificado anteriormente, para desenvolvimento do modelo de simulação seguiu-se os passos apresentados no item 3.2. No primeiro passo ficou definido que o problema em questão era a falta de um sistema adequado de programação da produção para o frigorífico estudado, dada a complexidade associada à variabilidade da matéria-prima e à sazonalidade das vendas. Não era raro faltar produto por problemas com a programação.

No segundo passo, definiu-se como meta do estudo, desenvolver um modelo de simulação para auxiliar à tomada de decisões sobre a programação da produção do frigorífico. Foi definido o cronograma de desenvolvimento do trabalho, o software de desenvolvimento do modelo de simulação e as formas de coleta de dados para identificar os pontos nevrálgicos do sistema/problema. O software de simulação utilizado foi o Arena, pois comporta as características presentes no frigorífico, quanto à aleatoriedade associada à matéria-prima e às vendas, sendo possível à análise dinâmica do sistema produtivo utilizando variáveis de estado determinísticas e estocásticas. 
No terceiro passo conceitualizou-se o modelo de acordo com as características do sistema utilizando a modelagem discreta, estocástica e dinâmica, composta de uma parte lógica, da qual são gerados os dados para análise estatística, e outra com uma animação para melhor visualizar o fluxo do processo e os resultados das variáveis durante a simulação.

A coleta de dados foi realizada durante o mês de janeiro de 2010. Apesar de tratar-se de um período pequeno e determinado do ano, lembrando que a empresa trabalha com demanda sazonal, os dados coletados foram suficientes para municiar o modelo. Algumas situações, não contempladas com os dados deste mês, foram parametrizadas e, assim, puderam também ser estudadas. Informações sobre o processo foram coletadas por meio de observação direta e de entrevista com o responsável da produção, enquanto que as informações sobre vendas e produção diária foram examinadas a partir dos registros históricos.

Terminada a coleta de dados, os processos de produção do frigorífico foram traduzidos para módulos no software Arena. Houve a preocupação em atender as especificidades de cada centro de produção, de modo que o conjunto funcionasse como o esperado. Na sequência foi feita a verificação do modelo, testando-o em relação às variáveis de controle. Um desses testes consistiu em zerar todos os estoques internos e habilitar apenas uma chegada de matéria-prima de 108 caixas. Com estas informações, o modelo foi executado e ao término da simulação obtiveramse resultados coerentes com o esperado, como pode ser observado na Tabela 1. A pequena porcentagem de erro resultante é devido à variabilidade dos pesos dos filés, sendo que a variável é composta pela média dos tempos padrões.

Tabela 1 - Porcentagens esperadas e produzidas

\begin{tabular}{lcccc}
\hline Subprodutos & Porcentagem & $\begin{array}{c}\text { Quantidade } \\
\text { esperada }\end{array}$ & $\begin{array}{c}\text { Quantidade } \\
\text { produzida }\end{array}$ & $\%$ Erro \\
\hline Filé & $32 \%$ & 691,2 & 699,84 & $1,25 \%$ \\
Couro & $3,30 \%$ & 71,28 & 72 & $1,01 \%$ \\
Carcaça e & $64,40 \%$ & 1391,04 & 1406,97 & $1,15 \%$ \\
vísceras & $0,30 \%$ & 6,48 & 6,56 & $1,23 \%$ \\
Espinho & $100 \%$ & 2160 & 2160 & $0,00 \%$ \\
\hline
\end{tabular}

Revista Produção Online, Florianópolis, SC, v.12, n. 4, p. 972-1001, out./dez. 2012. 
Para validar o modelo utilizaram-se dados históricos de produção e de venda das primeiras semanas de Janeiro. Após a inserção destes dados e execução do modelo, foi possível observar (Tabela 2) uma boa representação dos dados gerados pelo modelo em relação aos dados reais, resultando em pequenas porcentagens de diferença. Sendo assim, o modelo foi considerado validado.

Tabela 2 - Resultados da simulação comparados aos reais ${ }^{1}$

\begin{tabular}{|c|c|c|c|c|c|}
\hline \multicolumn{2}{|c|}{ Beneficiamento (kg) } & \multicolumn{2}{|c|}{ Embalagem (kg) } & \multicolumn{2}{|c|}{ Vendas (kg) } \\
\hline Real & Simulado & Real & Simulado & Real & Simulado \\
\hline 659 & 990 & 660 & 540 & 209 & 270 \\
\hline 656 & 950 & 633 & 1110 & 254 & 195 \\
\hline 513 & 957 & 515 & 985 & 1766 & 1845 \\
\hline 708 & 935 & 300 & 992 & 2249 & 2295 \\
\hline 779 & 989 & 930 & 975 & 30 & 16 \\
\hline 666 & & 810 & & & \\
\hline 637 & & 741 & & & \\
\hline 4618 & 4821 & 4589 & 4602 & 4508 & 4621 \\
\hline \multicolumn{2}{|c|}{$4 \%$} & \multicolumn{2}{|c|}{$0,28 \%$} & \multicolumn{2}{|c|}{$2,51 \%$} \\
\hline
\end{tabular}

No projeto de experimentos, o objetivo foi verificar a variabilidade dos dados de modo a averiguar se são confiáveis e, também, obter maior conhecimento sobre o sistema estudado e seus processos críticos. Para isso, identificaram-se as variáveis importantes de análise - variáveis controláveis e de resposta (Figura 4).

\footnotetext{
${ }^{1}$ As quantidades geradas no sistema real são diferentes das geradas pelo modelo de simulação, pois dependendo do dia, há até duas chegadas de matéria-prima na empresa, enquanto que no modelo isso é padronizado. Como o modelo simula a produção de cinco dias por semana, ele gerará apenas cinco resultados.

Revista Produção Online, Florianópolis, SC, v.12, n. 4, p. 972-1001, out./dez. 2012.
} 
Figura 4 - Variáveis estabelecidas do sistema

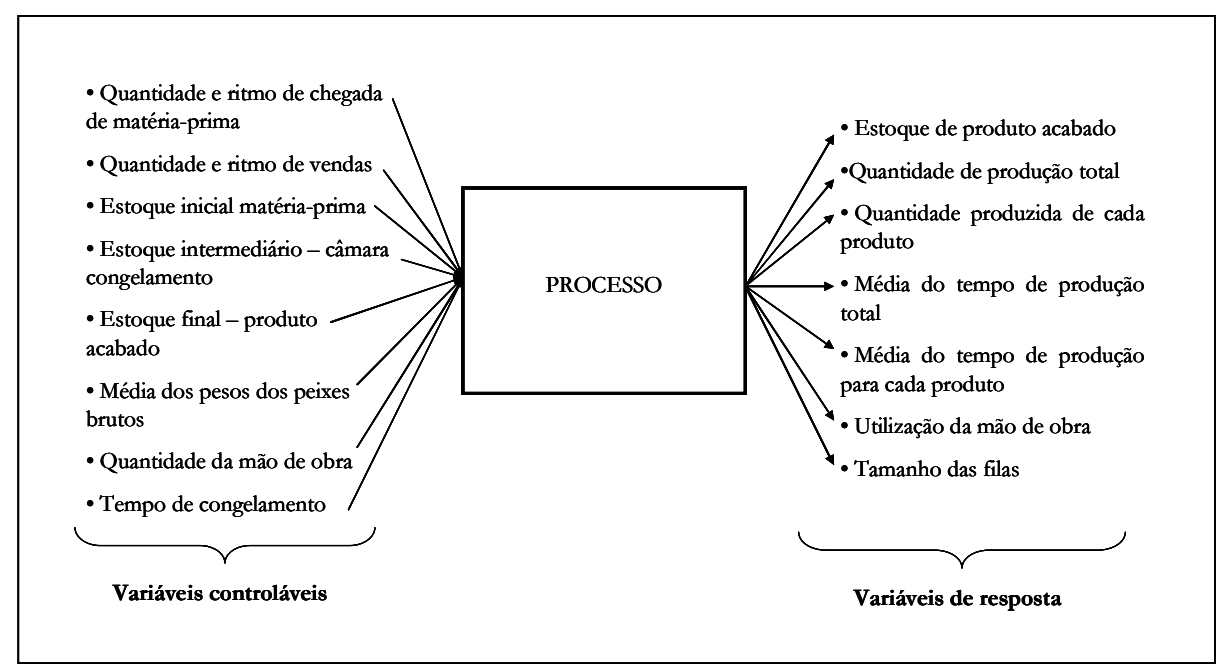

Definido as variáveis, executou-se o modelo por um período relativamente longo (2 meses) para se ter uma visão mais clara sobre o comportamento da variável "ESTOQUE_KG_FINAL" (estoque de produto acabado em quilogramas) no sistema e identificar o período que a mesma entra em regime (período transiente). Com auxílio do Output Analyser identificou-se um período transiente de 30260 segundos quando se atinge um valor no estoque de $2295 \mathrm{~kg}$. Como um período transiente resultante curto, aplicou-se diretamente o método das múltiplas replicações independentes, em que o modelo foi executado novamente com 20 replicações. Ao fim da simulação, calculou-se o intervalo de confiança da variável em questão, obtendo uma média de $4070 \mathrm{~kg}$ e uma half-width (h) de 55,9. Desejando-se 95\% de confiança nesta estatística, o half-width teria de ser no máximo 203,5; porém, com 20 replicações conseguiu-se um "h" bem inferior ao permitido. Portanto, conclui-se que os dados coletados e suas variabilidades durante o processo têm sustentação estatística.

Com o modelo pronto - verificado, validado e dentro dos padrões estatísticos -, demais simulações foram executadas para analisar os resultados e gerar estratégias de cenários adequados à produção. Os resultados das simulações são apresentados na Tabela 3, em que SR corresponde aos dados do sistema real, T1 e T2 são cenários propostos pelo OptQuest e o T3 e T4 são cenários sugeridos como tentativa de melhoria dos anteriores. O OptQuest é uma ferramenta de otimização integrada ao 
software Arena e tem a missão de simplificar a busca exaustiva de soluções, considerando todas as possíveis combinações entre fatores e indicando caminhos que gerem resultados melhores.

Tabela 3 - Resultados dos testes realizados ${ }^{2}$

\begin{tabular}{|c|c|c|c|c|c|c|c|c|c|c|}
\hline Colaboradores & Quant. & SR & Quant. & T1 & Quant. & T2 & Quant. & T3 & Quant. & T4 \\
\hline Colaborador LE & 2 & 0,0892 & 1 & 0,1788 & 1 & 0,1793 & 1 & 0,1792 & 1 & 0,1798 \\
\hline Colaborador F & 4 & 0,9537 & 1 & 0,9885 & 8 & 0,4758 & 5 & 0,7621 & 5 & 0,7623 \\
\hline Colaborador TC & 1 & 0,3557 & 1 & 0,0920 & 1 & 0,3555 & 1 & 0,3553 & 1 & 0,3555 \\
\hline Colaborador TSC & 2 & 0,4484 & 1 & 0,2328 & 2 & 0,4481 & 1 & 0,8956 & 2 & 0,4480 \\
\hline Colaborador TV & 2 & 0,7248 & 1 & 0,3759 & 2 & 0,7248 & 2 & 0,7249 & 2 & 0,7249 \\
\hline Colaborador C & 1 & 0,9256 & 2 & 0,1198 & 1 & 0,9257 & 2 & 0,4632 & 2 & 0,4624 \\
\hline Colaborador CB & 2 & 0,4995 & 1 & 0,2575 & 2 & 0,4997 & 2 & 0,4996 & 2 & 0,4993 \\
\hline Colaborador E & 1 & 0,9449 & 1 & 0,2795 & 2 & 0,5219 & 2 & 0,5314 & 2 & 0,5386 \\
\hline Tempo de sistema (h) & & 6,75 & & 8,05 & & 7,25 & & 5,87 & & 4,83 \\
\hline Produção Kg/semana & & 4701 & & 1220 & & 5100 & & 5240 & & 5405 \\
\hline Venda & & 5076 & & 4536 & & 4313 & & 4416 & & 4490 \\
\hline Estoque Final & & -376 & & -3316 & & 787 & & 824 & & 915 \\
\hline Processo & \multicolumn{10}{|c|}{ Filas } \\
\hline Lavar e escamar & & 0,3554 & & 0,1019 & & 0,1479 & & 0,1681 & & 0,1519 \\
\hline Filetar & & 61,4048 & & 25,0698 & & 32,6738 & & 46,4133 & & 46,0711 \\
\hline Tirar couro do filé & & 13,1421 & & 2,3183 & & 1,547 & & 7,0579 & & 7,1431 \\
\hline Tirar sobras couro & & 0,2376 & & 0,0904 & & 2,0298 & & 832,55 & & 0,2766 \\
\hline Tirar vezinho & & 0,6967 & & 0,1090 & & 650,69 & & 1,0273 & & 1,7556 \\
\hline Classificar & & 3,9583 & & 0,0695 & & 1341,34 & & 0,8101 & & 0,2788 \\
\hline Colocar bandeja & & 0,0327 & & 0,0234 & & 0,0344 & & 0,0338 & & 0,0424 \\
\hline Embalar & & 2,8295 & & 0,508 & & 0,9162 & & 0,9666 & & 1,9757 \\
\hline
\end{tabular}

No cenário do sistema real (SR), puderam-se observar altas taxas de utilização dos colaboradores F, C e E, tempo de sistema (tempo médio de produção) de 6,75 horas e um estoque final negativo. Não houve nenhum acúmulo prejudicial de entidades nas filas, considerando que a atividade a qual apresentou um maior número foi a filetagem e este fator é devido ao transporte ser feito na proporção de uma caixa (contendo uma média de 27 peixes) por colaborador. Durante o processo de filetagem os peixes são mantidos cobertos com gelo para a conservação da carne.

\footnotetext{
${ }^{2}$ As siglas dos colaboradores correspondem aos processos listados na Tabela. Logo: Colaborador LE (lavar e escamar), Colaborador F (filetar), Colaborador TC (tirar couro do filé), Colaborador TSC (tirar sobras couro), Colaborador TV (tirar vezinho), Colaborador C (classificar), Colaborador CB (colocar bandeja) e Colaborador E (embalar).

Revista Produção Online, Florianópolis, SC, v.12, n. 4, p. 972-1001, out./dez. 2012.
} 
Cinquenta simulações, com três replicações cada uma, foram executadas no OptQuest. Como restrição considerou-se que a soma das quantidades de colaboradores fosse menor ou igual a 36 (capacidade máxima) e como função objetivo, a redução do tempo de sistema, de forma a conseguir uma produção diária maior.

$\mathrm{O}$ teste 1 (T1) resultou em um montante de estoque negativo ainda maior que $\mathrm{O}$ sistema real simulado, apresentando, também, alta utilização do colaborador $F$, baixas taxas dos demais colaboradores e um aumento do tempo de sistema. Logo, considerou-se que esta estrutura de cenário não é apropriada.

No teste 2 (T2) dobrou-se a quantidade de colaborador $F$, em relação ao sistema real simulado, o que ocasionou um gargalo nas atividades de tirar o "vezinho" e classificar. Apesar de o estoque final ter fechado a semana com saldo positivo, as filas tornaram-se demasiadamente grandes (o que prejudica a qualidade do produto em permanecer muito tempo em espera no processo) e o tempo de sistema ainda permanece maior que do sistema real. Deste modo, tal estrutura não se encaixa no propósito desejado.

No teste 3 (T3) tentou-se balancear a linha de produção com base nas análises dos testes anteriores e dos tempos médios das atividades do processo. Como resultado, ainda obteve-se uma alta taxa de utilização do colaborador TSC, ocasionando, por conseqüência, um gargalo em sua atividade.

Finalmente, alterando a quantidade de recurso da atividade TSC, o teste 4 (T4) dispõe de um bom cenário para a produção demandada desta primeira semana de Janeiro. Alguns colaboradores ainda permaneceram com uma taxa de utilização baixa, contudo no caso das atividades de lavagem e retirada do couro é necessário a presença de pelo menos um colaborador em cada posto devido à distância deles dos demais postos. Já, no caso dos colaboradores TSC, C, CB e E as taxas de utilização estiveram em torno de 44 a $53 \%$, o que apesar de não ser um índice bom, excederia os $75 \%$ estabelecido com limitante, caso algum colaborador fosse retirado, e criaria gargalos por todo o processo. Ainda assim, com estas taxas baixas, conseguiu-se reduzir em $1,92 \mathrm{~h}$ do tempo de sistema e aumentar a produção em $704 \mathrm{~kg}$, cumprindo todas as solicitações de venda e permanecendo com um estoque de segurança. 
Importante ressaltar que em nenhum momento a variável custo foi critério de análise, pois o objetivo deste trabalho é utilizar a simulação como uma ferramenta útil a programação da produção, podendo visualizar a alocação dos recursos, a dinâmica dos fluxos e os tempos de processo.

Mesmo com o T4 obtendo o tempo médio de 4,83h para produção de uma caixa de $15 \mathrm{~kg}$ de filé de tilápia, foi possível reduzir-lo alterando o tempo de congelamento dos filés. Os tempos de 2 horas para congelamento dos filés frescos e 1 hora para 0 processo de glasing foram padronizados pela empresa sem estudos prévios, apenas pela observação e experiência diária. Assim, para encontrar o tempo ideal de congelamento dos filés frescos e dos filés após o processo de glasing, efetuaram-se cálculos utilizando conceitos teóricos e fórmulas específicas para congelamento de alimentos perecíveis, determinando um tempo de congelamento dos filés frescos de 5640 segundos (aproximadamente 94 minutos) e de permanência na câmara fria após o processo de glasing de 1440 segundos (aproximadamente 24 minutos). Com a alteração desses tempos no modelo, novo teste foi feito para verificar se houve alguma melhoria no processo. Pôde-se, então, verificar uma redução total no tempo de congelamento de $2,87 \mathrm{~h}$ se comparada ao sistema real. Em termos de taxa de utilização e número de filas, nada se alterou. A produção total também não teve grande aumento, pois toda a matéria-prima que entrou foi processada, sem restar nada na câmara de congelamento.

Finalizado o último passo do processo de desenvolvimento de um modelo de simulação, efetuou-se, também, a última etapa da metodologia SimuCAD - modelo final. O cenário que melhor se adaptou as variáveis iniciais estabelecidas foi o T4 com as mudanças no tempo de congelamento. O cenário final é mostrado na Figura 5 , onde é possível visualizar: a chegada de matéria-prima, as quantidades de caixas de peixe bruto e limpo, os colaborados ocupados ou inativos, a quantidade de carrinhos contidos na câmara de congelamento e as quantidades produzidas. Tudo isso de forma dinâmica, no decorrer da simulação dos cinco dias da semana. 
Figura 5 - Animação do modelo final $\left.\right|^{3}$

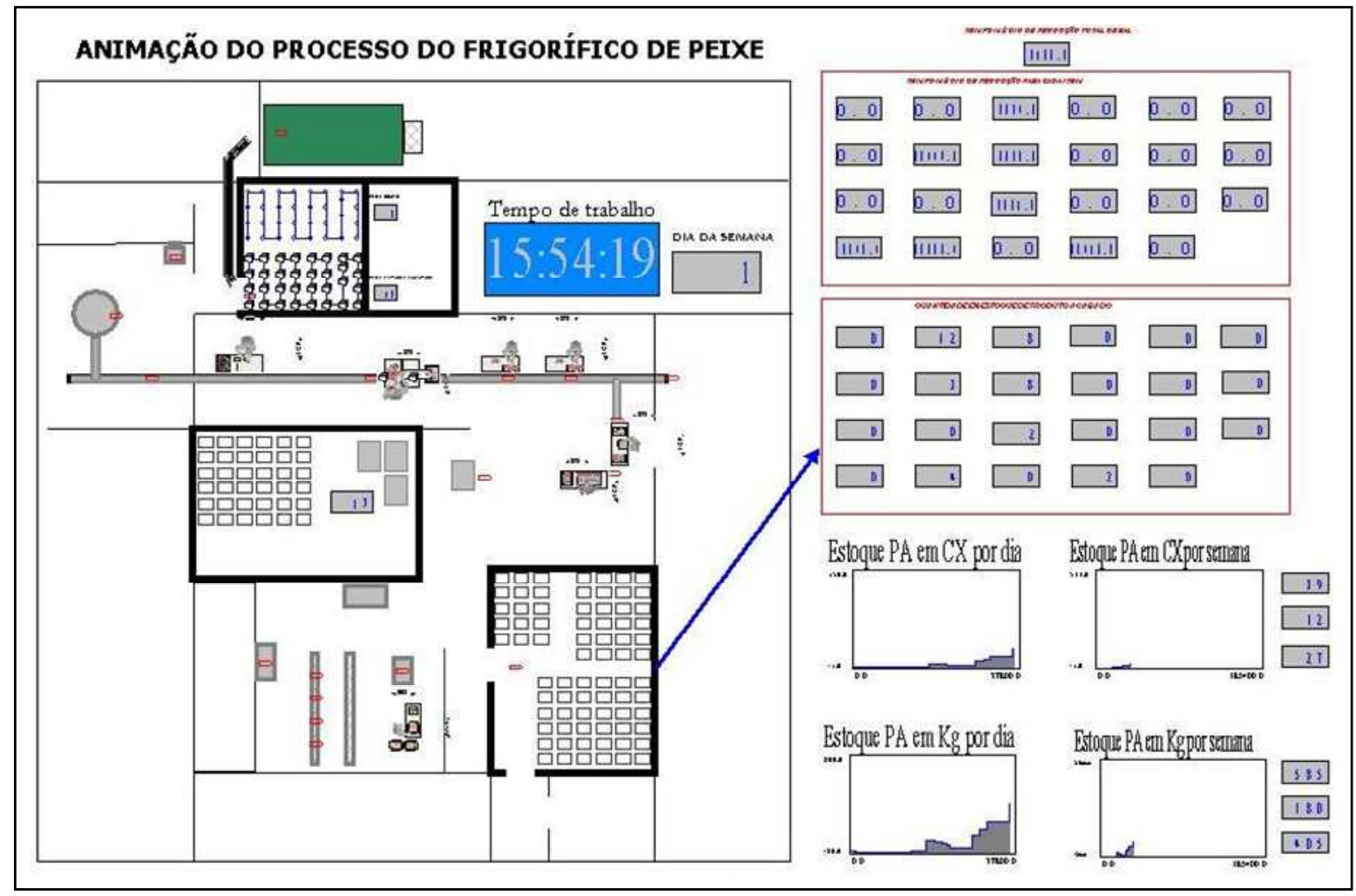

\subsection{Análise Geral dos Resultados}

Um primeiro ponto que pode ser melhorado é a eficiência dos funcionários da embalagem. Apesar da redução do tempo de congelamento, eles ainda possuem uma eficiência de 53,79\%. Para balancear esta etapa é possível reduzir ainda mais o tempo de congelamento dos filés modificando o mecanismo de congelamento para o Plate Freezers ou o Liquid imersion freezers (CLELAND; VALENTAS, 1997). Contudo de modo a causar um menor impacto possível na estrutura existente e ter baixos investimentos, é possível reduzir o tempo de congelamento dos filés frescos para 59 minutos, em média, aumentando apenas a velocidade do ventilador da câmara de 2 $\mathrm{m} / \mathrm{s}$ para $4 \mathrm{~m} / \mathrm{s}$.

A tríade piscicultura/frigorífico/distribuidores possui grandes vantagens com um relacionamento próximo. Começando pela piscicultura (fornecedora de tilápias), a

\footnotetext{
${ }^{3}$ Figura extraída da animação desenvolvida no software Arena.

Revista Produção Online, Florianópolis, SC, v.12, n. 4, p. 972-1001, out./dez. 2012.
} 
mesma desfruta de grandes vantagens perante o seu modo de cultivo em tanques-rede e pela pequena distância até o frigorífico. Este, por sua vez, usufrui do crescimento do mercado e produz, cada vez mais, para atender a demanda. Por fim, os distribuidores (responsáveis pelas maiores vendas do frigorífico) abastecem diferentes regiões onde há demanda. Neste processo de relacionamento é preciso atenção quanto: à execução de uma boa programação da produção no curto prazo, além do desenvolvimento de um planejamento da produção em longo prazo; à gestão da cadeia de suprimentos e da demanda que não existe no momento devido à ausência de histórico e a falta de conhecimento dos gestores desta cadeia para execução de uma previsão da demanda robusta (PEREIRA et al., 2010b); e ao aproveitamento dos resíduos do processo de modo a não prejudicar o meio ambiente e, por conseguinte, a sociedade (PEREIRA; COSTA; NOGUEIRA, 2010a; KUBITZA, 2005).

O principal desafio de um sistema de planejamento e controle da produção é o balanceamento entre a oferta e a procura, sendo ambas dimensões de alta variabilidade. Assim, combiná-las é um desafio permanente da gerência de produção. A previsão de vendas facilita a programação da produção e, consequentemente, a elaboração dos planos de produção com antecedência, tomando medidas preventivas. Logo, para a obtenção de um ótimo planejamento da produção é essencial que - não só esta empresa, mas todas em geral - trabalhem com uma boa base em suas previsões de venda, efetuando uma gestão da demanda no decorrer dos planos de longo, médio e curto prazo.

A sazonalidade também é um ponto influente na programação da produção do frigorífico. De acordo com Slack, Chambers e Johnston (2002), são seis as causas da sazonalidade: climáticas, festivas, comportamentais, políticas, financeiras e sociais. No caso do frigorífico as causas sazonais são as climáticas e as festivas. O verão é considerado uma causa climática, pois é nesta época que há um maior desenvolvimento dos peixes e, também, maior venda em decorrência da mudança de hábitos alimentares dos consumidores locais e regionais nesta época (férias). Como causas festivas, têm-se as festas de final de ano (Reveillon e Natal), nas quais além de carnes vermelhas e aves, já se encontram cardápios repletos de peixes, e a quaresma, 
na qual é tradição do catolicismo o não consumo de carnes vermelhas, proporcionando um aumento no consumo de peixes.

\section{CONCLUSÃO}

A perspectiva deste estudo foi abordar a simulação computacional para auxílio à programação da produção de um frigorífico de peixe específico. Programar a produção é uma atividade bastante complexa, pois envolve tempo de produção, prazo de entrega, mix de produto, quanto, quando e onde produzir, além de buscar sempre o equilíbrio entre capacidade produtiva e demanda. E com o objetivo de reduzir as dificuldades de programação é que as empresas estão, cada vez mais, fazendo uso de sistemas computacionais, softwares especializados e simuladores para o gerenciamento de seus sistemas produtivos, dispondo de soluções avançadas, com mais rapidez e segurança do que a concorrência.

O modelo de simulação desenvolvido respondeu aos objetivos estabelecidos, gerando resultados confiáveis e úteis para auxiliar na tomada de decisões sobre a programação da produção do frigorífico em estudo. Este modelo também pode ser empregado em outras empresas com processos produtivos análogos ao estudado. Tendo por base o referencial teórico elaborado, a pesquisa desenvolvida e o objetivo proposto foi possível: gerar cenários de produção mais confiáveis; testar diversas variáveis de controle; aproveitar melhor os recursos e quantificá-los; obter planos de produção semanais ou diários; verificar a capacidade do sistema; analisar o estado dos estoques; e determinar a quantidade de matéria-prima necessária. Tudo isso identificando se será necessário adquirir mais mão de obra, fazer horas extras, utilizar os colaboradores em tempo parcial, ou seja, identificar através dos resultados gerados pela simulação um método de ajuste da capacidade. Tais decisões afetam os custos, as receitas, o capital de giro, a qualidade, a velocidade, a confiabilidade e a flexibilidade do sistema produtivo. Diante deste contexto, tomadas de decisão através das informações geradas pelo modelo de simulação aqui construído podem atender bem as expectativas de desempenho da empresa. 
Além destes pontos, que são fundamentais para uma boa programação da produção, conseguiu-se reduzir o lead-time do processo de transformação dos produtos, por meio de cálculo específicos do tempo de congelamento dos filés frescos e do processo de glasing, e visualizar as consequências benéficas geradas.

Também, é relevante enfatizar que o investimento em estudos e pesquisas no ramo de processamento de peixes é promissor, pois o consumo mundial per capita de pescados tem aumentado muito em virtude das mudanças alimentares e dos incentivos a aquicultura (FIGUEIREDO JUNIOR; VALENTE JUNIOR, 2008). Por conta deste crescimento, unidades de processamento de tilápias estão surgindo cada dia mais. Tal destaque nesta espécie é devido a determinadas características como: pequeno ciclo de engorda; desova durante todo o ano; resistência a doenças, altas densidades de povoamento e a baixos níveis de oxigênio dissolvido; boas características organolépticas; e excelente rendimento de filé (HILSDORF, 1995).

Portanto, a simulação foi considerada, neste caso, uma boa técnica para tratar o problema de programação exposto, tendo como vantagem a visualização do funcionamento do frigorífico de forma rápida e a baixo custo. Tal ferramenta tem seu valor aumentado ao aliar-se com outras técnicas ou estudos aprofundados sobre melhoria do processo, como ocorreu com o estudo dos tempos de congelamento seguido de uma posterior simulação.

Possíveis trabalhos futuros para complementar o presente trabalho e ampliar 0 conhecimento neste setor emergente podem ser: inclusão da previsão e gestão da demanda como forma de ampliar a certeza sobre os resultados gerados pelo modelo de simulação desenvolvido; desenvolvimento de um planejamento da produção incluindo os três níveis hierárquicos; e análise do processo de glasing, suas vantagens e desvantagens, identificando se este processo gera ou não margem de lucro para a empresa.

Nota:

Texto inédito, fruto de uma dissertação desenvolvida pela autora, financiada pela Capes, e orientada pelo co-autor por meio do Programa de Pós-Graduação em Engenharia de Produção da Universidade Federal de São Carlos. 


\section{REFERÊNCIAS}

ALEXANDER, C.W. Discrete event simulation for batch processing. In: WINTER SIMULATION CONFERENCE, 2006, Augusta. Anais... Augusta: Lovers Lane, p. 19291934, 2006.

BANKS, J. Principles of simulation. In: Handbook of simulation: principles, methodology, advances, applications and practice. Nova York: John Wiley \& Sons, 1998.

BOIKO, T.J.P.; TSUJIGUCHI, L.T.A.; VAROLO, F.W.R. Classificação de Sistemas de Produção: uma abordagem de Engenharia de Produção. In: ENCONTRO DE PRODUÇÃO CIENTÍFICA E TECNOLÓGICA, 4., 2009. Campo Mourão, Anais... Campo Mourão: NUPEM, 2009.

BROSCOLO, W.R., et al. Desempenho e características de carcaça de machos revertidos de tilápias do Nilo (Oreochromis niloticus), linhagens tailandesa e comum, nas fases inicial e de crescimento. Revista Brasileira de Zootecnia, v.30, n.5, p. 13911396, 2001.

CAUCHICK MIGUEL, P.A.C., et al. Metodologia de pesquisa em engenharia de produção e gestão de operações. Rio de Janeiro: Elsevier, 2010.

CHASE, R.B.; JACOBS, F.R.; AQUILANO, N.J. Administração da produção para a vantagem competitiva. 10. ed. São Paulo: Bookman, 2006.

CLELAND, D.J.; VALENTAS, K.J. Prediction of Freezing Time and Design of Food Freezers. In: VALENTAS, K.J.; ROTSTEIN, E.; SINGH, R.P. Handbook of Food Engineering Practice. Nova York: CRC Press, 1997.

CONTADOR, J.C.; CONTADOR, J.L. Programação e controle da produção para a indústria intermitente. In: CONTADOR, J. C. (coordenador). Gestão de Operações. 2 ed. São Paulo: Fundação C. A. Vanzolini; Edgard Blücher, 1997.

COSTA, M.A.B. Simulação de sistemas. Apostila de suporte a disciplina Simulação aplicada a Engenharia de Produção. Departamento de Engenharia de Produção. 2002.

COSTA, M.A.B. Um modelo baseado em conhecimento para simular rebanhos de bovinos de corte. 2004. 188 p. Tese (Doutorado em Engenharia Elétrica e de Computação) - Faculdade de Engenharia Elétrica e de Computação, Universidade Estadual de Campinas, Campinas, 2004.

COSTA, M.A.B.; MENEGON, N.L.; CAMAROTTO, J.A. SimuCAD: Simulação \& CAD um sistema de apoio à decisão no planejamento e implantação de sistemas de 
produção. In: ENCONTRO NACIONAL DE ENGENHARIA DE PRODUÇÃO, 16., 1996, Piracicaba. Anais... Piracicaba: ABEPRO, 1996.

DIRENE, J.R. Análise de planejamento e controle da produção em uma empresa do ramo rural. 2003. 91 p. Monografia (Graduação em Engenharia de Produção), Universidade Federal de Ouro Preto, Ouro Preto, 2003.

DUARTE, R.N. Simulação computacional: análise de uma célula de manufatura em lotes do setor de auto-peças. 2003. 235 p. Dissertação (Mestrado em Engenharia de Produção) - Programa de Pós-Graduação em Engenharia de Produção, Universidade Federal de Itajubá, Itajubá, 2003.

FERNANDES, F.C.F; GODINHO FILHO, M. Planejamento e controle da produção, dos fundamentos ao essencial. São Paulo: Atlas, 2010.

FIGUEIREDO JUNIOR, C.A.; VALENTE JUNIOR, A.S. Cultivo de tilápia no Brasil: origens e cenário atual. In: CONGRESSO DA SOCIEDADE BRASILEIRA DE ECONOMIA, ADMINISTRAÇÃO E SOCIOLOGIA RURAL, 47., 2008, Fortaleza. Anais... Rio Branco: SOBER, 2008.

FREITAS FILHO, P.J. Introdução à modelagem e simulação de sistemas: com aplicações em Arena. Florianópolis: Visual Books, 2001.

GAVIRA, M.O. Simulação computacional como uma ferramenta de aquisição de conhecimento. 2003. Dissertação (Mestrado em Engenharia de Produção) - Escola de Engenharia de São Carlos, Universidade de São Paulo, São Carlos, 2003.

GIL, A.C. Métodos e técnicas de pesquisa social. 5. ed. São Paulo: Atlas, 1999.

HARREL, C.H.; TUMAY, K. Simulation made easy: a manager's guide. Engineering and Norcross; Management Press, 1995.

HARRINGTON, H.J.; TUMAY, K. Simulation modeling methods. Nova York: McGrawHill, 2000.

HILSDORF, A.W.S. Genética e cultivo de tilápias vermelhas: uma revisão. Boletim do Instituto de Pesca, São Paulo, v. 22, n. 1, p. 73-84, 1995.

IANNONI, A.P.; MORABITO, R. Análise do sistema logístico de recepção de cana-de-açúcar: um estudo de caso utilizando simulação discreta. Gestão \& Produção, v.9, n.2, p.107-128, 2002.

JENSSON, P. Daily production planning in fish processing firms. European Journal of Operational Research, v.36, p.410-415, 1988. 
JONATANSON, E.; RANDHAWA, S.U. A simulation based decision model for a fish processing facility. Computers Industrial Engineering, v.11, p.406-410, 1986.

JORY, D.E.; ALCESTE, C.; CABRERA, T.R. Mercado y comercialización de tilapia en los Estados Unidos de Norteamérica. Panorama Acuícola, v.5, n.5, p.50-53, 2000.

KELTON, D.; SADOWSKI, D. A.; SADOWSKI, R. P. Simulation with Arena. 1 ed. New York: McGraw-Hill, 1998.

KELTON, W.D.; SADOWSKI, R.P.; SWETS, N.B. Simulation with Arena. 5 ed. New York: McGraw-Hill, 2010.

KUBITZA, F. Coletânea de informações aplicadas ao cultivo de tambaqui, do pacu e outros peixes redondos - Parte 2. Revista Panorama da Aqüicultura, São Paulo, v.14, n.83, p.13-23, maio/jun. 2004.

KUBITZA, F. Tilápia em água salobra e salgada: uma boa alternativa de cultivo para estuários e viveiros litorâneos. Revista Panorama da Aqüicultura. São Paulo, v.15, n.88, p.14-18, 2005.

KUBITZA, F. Tilápias na Bola de Cristal. Revista Panorama da Aquicultura. São Paulo, v.17, n.99, jan./fev. 2007.

LAW, A.M.; KELTON, W.D. Simulation modeling and analysis. 3. ed. Nova York: McGraw Hill, 2000.

LI, J.; MEERKOV, S.M.; ZHANG, L. Production systems engineering: Problems, solutions, and applications. Annual Reviews in Control, v.34, p.73-88, 2010.

LOBÃO, E.C.; PORTO, A.J.V. Proposta de sistematização de estudos de simulação. Revista de Engenharia e Arquitetura - EESC/USP, São Carlos, SP, p. 61-69, 1996.

LÖFGREN, B.; TILLMAN, A.M. Relating manufacturing system configuration to life-cycle environmental performance: discrete-event simulation supplemented with LCA. Journal of Cleaner Production, v.19, p. 2015-2024, 2011.

MEDAU, J.C. Análise de capacidade do lado aéreo de aeroportos baseada em simulação computacional: aplicação ao aeroporto de São Paulo - Congonhas. 2011. 123p. Dissertação (Mestrado em Engenharia de Transportes) - Programa de PósGraduação em Engenharia de Transportes, Escola Politécnica da Universidade de São Paulo, 2011.

MORABITO, R.; PUREZA, V. Modelagem e simulação. In: CAUCHICK MIGUEL, P.A.C. et al. Metodologia de pesquisa em engenharia de produção e gestão de operações. Rio de Janeiro: Elsevier, 2010. p.165-192. 
NUNES, D.M.; MELO, P.A.C.; NIGRO, I.S.C. Planejamento, programação e controle da produção: o uso da simulação do PREACTOR em uma indústria de alimentos. In: Congresso Nacional de Engenharia de Produção, 29, 2009, Salvador. Anais... Salvador, ENEGEP: 2009.

OLIVEIRA, C.S. Aplicação de técnicas de simulação em projetos de manufatura enxuta. Estudos Tecnológicos, v.4, n.3, p. 204-217, 2008.

ÖREN, T.I. Simulation and reality: The big picture. International Journal of Modeling, Simulation, and Scientific Computing, v.1, p.1-25, 2010.

ÖREN, T.; YILMAZ, L. Synergies of simulation, agents, and systems engineering. Expert Systems with Applications, v.39, p.81-88, 2012.

PAUL, R.J.; KULJIS, J. Problem solving, model solving, or what? In: WINTER SIMULATION CONFERENCE, 2010, Baltimore. Anais... Baltimore: Lovers Lane, p. 353-358, 2010.

PEGDEN, C.D.; SHANNON, R.E.; SADOWSKI, R.P. Introduction to simulation Using SIMAN. 2. ed. New York: McGraw-Hill, 1995.

PEREIRA, C.R; COSTA, M.A.B; NOGUEIRA, E. Sustentabilidade Ambiental e Estratégia: estudo de caso em um frigorífico de peixe do interior do Estado de São Paulo. In: CONGRESSO NACIONAL DE ENGENHARIA DE PRODUÇÃO, 30, 2010, São Carlos. Anais... São Carlos: ABEPRO,2010a.

PEREIRA, C.R., et al. A Gestão da Demanda em uma Cadeia de Suprimentos Emergente: o caso de um frigorífico de peixe do estado de São Paulo. In: CONGRESSO NACIONAL DE ENGENHARIA DE PRODUÇÃO, 30, 2010, São Carlos. Anais... São Carlos: ABEPRO, 2010b.

RANDHAWA, S.U.; BJARNASON, E.T. A decision aid for coordinating fishing and fish processing. European Journal of Operational Research, v.81, p.62-75, 1995.

RESENDE, A.L.S.S. Viabilidade Técnica, Qualidade Nutricional e Sensorial de Produtos à Base de Carne de Tilápia (Oreochromis niloticus). 2010. $112 \mathrm{p}$. Tese (Doutorado em Ciência e Tecnologia de Alimentos) - Programa de Pós-Graduação em Ciência e Tecnologia de Alimentos, Universidade Federal Rural do Rio de Janeiro, Rio de Janeiro, 2010.

SHANNON, R.E. System simulation: the art and science. Englewood Cliffs, N.J: Prentice Hall, 1975. 
SHANNON, R.E. Introduction to the art and science of simulation. In: WINTER SIMULATION CONFERENCE, 1998, Texas. Anais... Texas: Texas A \& M University, 1998.

SLACK, N.; CHAMBERS, S.; JOHNSTON, R. Administração da Produção. 2 ed. São Paulo: Editora Atlas, 2002.

SIPPER, D.; BULFIN, R.L. Production: planning, control and integration. Nova York: McGraw-Hill, 1997.

SILVA, J.E.A.R. Desenvolvimento de um modelo de simulação para auxiliar o gerenciamento de sistemas de corte, carregamento e transporte de cana-deaçúcar. 2006. 145p. Dissertação (Mestrado em Engenharia de Produção) - Programa de Pós-Graduação em Engenharia de Produção, Universidade Federal de São Carlos. São Carlos, 2006.

SOARES, P. Secretaria faz campanha nacional para vender quilo de peixe a $R \$ 1,00$. Folha de São Paulo. São Paulo, 20 set. 2005.

SUSSEL, F.R. Para onde vai à tilápia: anuário da Pecuária Brasileira. In: ANUÁRIO DA PECUÁRIA BRASILEIRA, 2007, São Paulo. Anais... São Paulo: FNP, 2007.

TUBINO, D.F. Manual de planejamento e controle da produção. São Paulo: Atlas, 1997.

UM, I.; HYEONJAE, C.; LEE, H. The simulation design and analysis of a Flexible Manufacturing System with Automated Guided Vehicle System. Journal of Manufacturing Systems, v.28, p.115-122, 2009.

VOLLMANN, T. E.; BERRY, W. L.; WHYBARK, D.C; JACOBS, F. R. Manufacturing Planning and Control for Supply Chain Management. 5 ed. Boston: Mcgraw-Hill, 2004.

WANG, S.C. et al. Explore of the hospital management of simulation education learning model. Journal of Engineering Technology and Education, v.7, n.4, p.640-649, 2010.

WEI, J., LEUNG, S.C.H. A simulation modeling and analysis for RFID-enabled mixedproduct loading strategy for outbound logistics: A case study. Computers \& Industrial Engineering, v.61, p.209-215, 2011.

WY, J. et al. A data-driven generic simulation model for logistics-embedded assembly manufacturing lines. Computers \& Industrial Engineering, v.60, p.138-147, 2011.

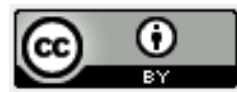

Artigo recebido em 27/04/2011 e aceito para publicação em 11/01/2012.

Revista Produção Online, Florianópolis, SC, v.12, n. 4, p. 972-1001, out./dez. 2012. 